ORIGINAL ARTICLE

\title{
Respiratory illness associated with influenza and respiratory syncytial virus infection
}

\author{
D M Fleming, R S Pannell, A J Elliot, K W Cross
}

See end of article for authors' affiliations

......................

Correspondence to: Dr D M Fleming,

Birmingham Research Unit of The Royal College of General Practitioners, Lordswood House, 54 Lordswood Road, Harborne, Birmingham B179DB, UK; dfleming@ rcgpbhamresunit.nhs.uk

Accepted

18 February 2005

Published Online First 26 April 2005

\begin{abstract}
Aims: To estimate excess morbidity during periods of influenza and respiratory syncytial virus (RSV) activity.

Methods: Retrospective analysis of a sentinel practice network database in active and non-active virus periods. Main outcome measures: clinical diagnoses of new episodes of influenza-like illness (ILI), acute bronchitis, asthma, and otitis media.

Results: The clinical diagnosis of ILI was consistent with influenza virus activity and acute bronchitis with RSV. During periods of virus activity, estimates of excess morbidity in children aged 1-4 and 5-14 years diagnosed as having acute otitis media exceeded those diagnosed with each of the other three conditions; in children $<1$ year estimates for acute bronchitis were highest. Using a broad definition of virus activity and summarising the data for all children diagnosed with ILI, $60 \%$ was attributable to influenza (40\% RSV) as were $37 \%$ of episodes diagnosed as acute bronchitis, $9 \%$ of those with asthma and $48 \%$ of those with otitis media. Using a narrow definition, corresponding proportions were: for ILI diagnoses $77 \%$ (23\% RSV), acute bronchitis $32 \%$, asthma zero, and otitis media $45 \%$. Acute bronchitis was diagnosed twice as frequently in association with RSV as with influenza in all age groups: excess asthma episodes were only evident in RSV active periods.

Conclusions: Except in relation to ILI, RSV caused more illness than the influenza virus in the respiratory diagnoses examined, emphasising the need for RSV prevention and treatment. Influenza was not associated with excess asthma episodes.
\end{abstract}

$P$ reventive and prophylactic programmes for disease management require an understanding of the natural history and complications associated with the disease. In the United Kingdom annual vaccination against influenza is recommended for children at risk because of co-morbidity including those with asthma, diabetes, chronic cardiovascular or renal disease, and the immune compromised: there is no recommendation for universal vaccination. The evidence base for this policy is derived mainly from extrapolating to children, the results of studies of vaccine efficacy in adults. ${ }^{12}$ Palivizumab (a monoclonal antibody) is available for prophylaxis against respiratory syncytial virus (RSV), but it is very expensive and has limited clinical application.

Influenza and RSV cause lower respiratory tract infections in persons of all ages, though RSV infection impacts particularly in young children. ${ }^{3}{ }^{4}$ Both viruses are prevalent in most winters, often at about the same time. Both mutate frequently and thus recurrent infections are common. Children infected with either virus commonly present with fever and cough, sometimes accompanied by wheeze, and some because of otitis media. ${ }^{5}$

RSV infection has been associated with the subsequent development of asthma, but conversely a lack of exposure to common virus infections has been suggested as a cause of asthma. ${ }^{6-9}$ The highest asthma attack rates in young children occur in December when the impact of RSV in the community is greatest. ${ }^{10}$ Many other viruses are known to be associated with asthma attacks. ${ }^{611-15}$

The diagnosis of asthma in young children is difficult: it is usually made clinically when the child is seen in an attack and is based on the clinical history and presence of bronchospasm. Acute bronchitis is diagnosed much more frequently than asthma. ${ }^{16}$ Otitis media can also be difficult to diagnose, especially in young children (because of the absence of direct communication and the difficulty of getting a good view of the eardrum). Incidence is strongly correlated at a weekly level with upper respiratory tract infections. ${ }^{17}$

We aimed to quantify the impact of influenza virus and RSV on clinical diagnoses of influenza-like illness (ILI), acute bronchitis, asthma, and otitis media by estimating the excess children reporting to general practitioners with these conditions during influenza and RSV active periods over the six winters $1994 / 95$ to $1999 / 2000$.

\section{METHODS}

The Weekly Returns Service (WRS) of the Royal College of General Practitioners has collected data on selected new episodes of illness since $1967 .{ }^{18}$ With increasing computerisation, data collection was extended in 1994 to include all diseases. General practitioners define the problems presented at each consultation in diagnostic terms where possible and distinguish between new episodes of illness and follow up consultations. Diagnosis is clinically based, though guidelines (not age specific) have been circulated to the recorders for selected conditions. As an example, the guidance for acute bronchitis (and bronchiolitis) specifies an acute illness with a cough and scattered or generalised abnormal chest signs including wheeze, and coarse or moist sounds. For asthma, a new episode is deemed to occur when a person consults because of an asthma attack or if his asthma is out of control. Weekly incidence rates (per 100000 persons) for new episodes of ILI, acute bronchitis, asthma, and otitis media were assembled for the six winters. These were grouped by age ( $<1,1-4,5-14$ years) and compared with viral isolates for influenza (A or B) and RSV confirmed cases reported to the Health Protection Agency (HPA).

Abbreviations: HPA, Health Protection Agency; ILI, influenza-like illness; RSV, respiratory syncytial virus; WRS, Weekly Returns Service 
Table 1 Influenza and RSV active periods by week number (weeks duration)

\begin{tabular}{|c|c|c|c|c|c|}
\hline \multicolumn{3}{|c|}{ Influenza ( $A$ and $B$ ) } & \multicolumn{3}{|l|}{ RSV } \\
\hline Year & Broad definition & Narrow definition & Year & Broad definition & Narrow definition \\
\hline $\begin{array}{l}1994 / 95 \\
1995 / 96 \\
1996 / 97 \\
1997 / 98 \\
1998 / 99 \\
1999 / 00\end{array}$ & $\begin{array}{l}1-14(14) \\
45-3(11) \\
49-9(13) \\
6-14(9) \\
51-7(9) \\
50-5(8)\end{array}$ & $\begin{array}{l}4-10(7) \\
47-1(7) \\
2-6(5) \\
9-15(7) \\
51-5(7) \\
51-3(5)\end{array}$ & $\begin{array}{l}1994 / 95 \\
1995 / 96 \\
1996 / 97 \\
1997 / 98 \\
1998 / 99 \\
1999 / 00\end{array}$ & $\begin{array}{l}45-4(12) \\
46-6(13) \\
47-9(15) \\
44-3(12) \\
45-3(11) \\
44-1(10)\end{array}$ & $\begin{array}{l}49-1(5) \\
50-4(7) \\
47-1(7) \\
48-52(5) \\
47-51(5) \\
46-50(5)\end{array}$ \\
\hline
\end{tabular}

For each winter, diagnosis, and age group, we calculated the mean weekly incidence rate in virus active periods and the excess over a baseline rate formed from the remaining winter weeks (week 40 of one year to week 20 of the next). This excess was multiplied by the number of weeks in which the virus was active to estimate the cumulative winter excess. The calculation was initially performed for a combined RSV and influenza active period, then repeated for the two viruses separately. This combined cumulative winter excess rate was allotted to RSV and influenza in the same proportion as the separate virus estimates.

Active periods were defined (broad definition) from the clinical incidence of ILI as previously described ${ }^{19}$ and from weekly RSV reports to the HPA exceeding 200 in children $<1$ year old. Week 52 was treated as active if reports in both weeks 51 and 01 exceeded 200, because laboratory investigation routines are limited during the Christmas and New Year period. The sensitivity of the definition of the active periods was explored in a narrow definition based solely on viral isolates (influenza) and virological reports (RSV). Examination of weekly virus counts revealed that, although the magnitude of peaks varied between age bands for both influenza and RSV, a similar time trend was evident in all ages. Hence, the active periods were defined by incorporating sufficient weeks, around the peak week to include $40 \%$ of all influenza isolates and 50\% of all RSV reports for the entire winter (table 1). This definition was chosen to provide maximum separation of influenza virus and RS virus active periods. Further definitions embracing greater proportions were explored. The relative proportions of excess diagnoses attributable to influenza and RSV were compared for each definition.

\section{RESULTS}

The cumulative population (person-weeks) on which this study is based is detailed in table 2. Periods of virus activity (influenza and RSV combined) and the respective populations are here defined by the broad definition. The narrow definition included 67 active weeks altogether; populations in virus active weeks were approximately one third less.
Weekly clinical incidence data for ILI in children in the three age groups during each winter are compared with virus report data in fig 1A. In the winters in which RSV and influenza virus were best separated (1994/95 and 1997/98), reported incidence of ILI was more clearly evident in the influenza active period than in the RSV active period. The same tendency, though less obvious, was observed in 1998/99 and 1999/00. In 1995/96 and 1996/97 there was very little differentiation between influenza and RSV active periods.

A similar comparison is made for acute bronchitis (fig 1B), which is reported five times as frequently as ILI. Clinical incidence aligns very closely with RSV active periods, though a secondary less obvious incidence surge is evident in those winters in which influenza follows RSV. The results for asthma (fig lC) are less clear; incidence surges were found in four of the six RSV active periods (exceptions 1995/96 and 1996/97). There were no particular surges during influenza virus activity, and surges were found when neither virus was circulating. The incidence of otitis media (fig lD) is evident over both RSV and influenza active periods.

Figure 1 also shows differences between age groups. For ILI, incidence rates were similar in all age groups. For acute bronchitis, incidence in children $<1$ year was much higher than that in older age groups, though for asthma it was less. In the age group 5-14 years, for acute bronchitis, asthma, and otitis media, incidence was less than in younger age groups: the impact during RSV periods was generally less than that seen in younger children.

Table 3 summarises the average weekly baseline incidence in non-virus active periods for each winter, diagnosis, and age group. For ILI, baseline rates were around 25 per 100 000: for otitis media and acute bronchitis, rates were much higher than those for ILI and asthma. For each of these diagnoses, rates were less in 1999/00 than in 1994/95. In children <1 year, background rates of asthma were about a tenth of those for acute bronchitis; in age group 1-4 years, half; and 5-14 years, roughly equal.

The cumulative winter excess rates of ILI, acute bronchitis, asthma, and otitis media reported during RSV and influenza active periods based on the broad definition are summarised for each winter in fig 2 . It shows considerable variation between

Table 2 Cumulative population (person-weeks) in combined influenza and RSV active period and number of active weeks according to broad definition

\begin{tabular}{|c|c|c|c|c|c|c|c|}
\hline \multirow[b]{2}{*}{ Year } & \multicolumn{3}{|c|}{ Active weeks } & \multicolumn{3}{|c|}{ Inactive weeks } & \multirow{2}{*}{$\begin{array}{l}\text { No. of active } \\
\text { weeks }\end{array}$} \\
\hline & $<1$ year & $1-4$ years & 5-14 years & $<1$ year & $1-4$ years & 5-14 years & \\
\hline $1994 / 95$ & 80491 & 393072 & 958342 & 41924 & 208262 & 509486 & 22 \\
\hline $1995 / 96$ & 55445 & 274699 & 685557 & 76674 & 376913 & 947064 & 14 \\
\hline $1996 / 97$ & 67700 & 313479 & 803187 & 82850 & 380626 & 982392 & 15 \\
\hline $1997 / 98$ & 98911 & 459805 & 1237457 & 55730 & 261737 & 703763 & 21 \\
\hline $1998 / 99$ & 80727 & 366577 & 995012 & 101877 & 457277 & 1249411 & 15 \\
\hline $1999 / 00$ & 75169 & 349196 & 955037 & 109955 & 511910 & 1403246 & 14 \\
\hline Total & 458443 & 2156828 & 5634592 & 469010 & 2196725 & 5795362 & 101 \\
\hline
\end{tabular}




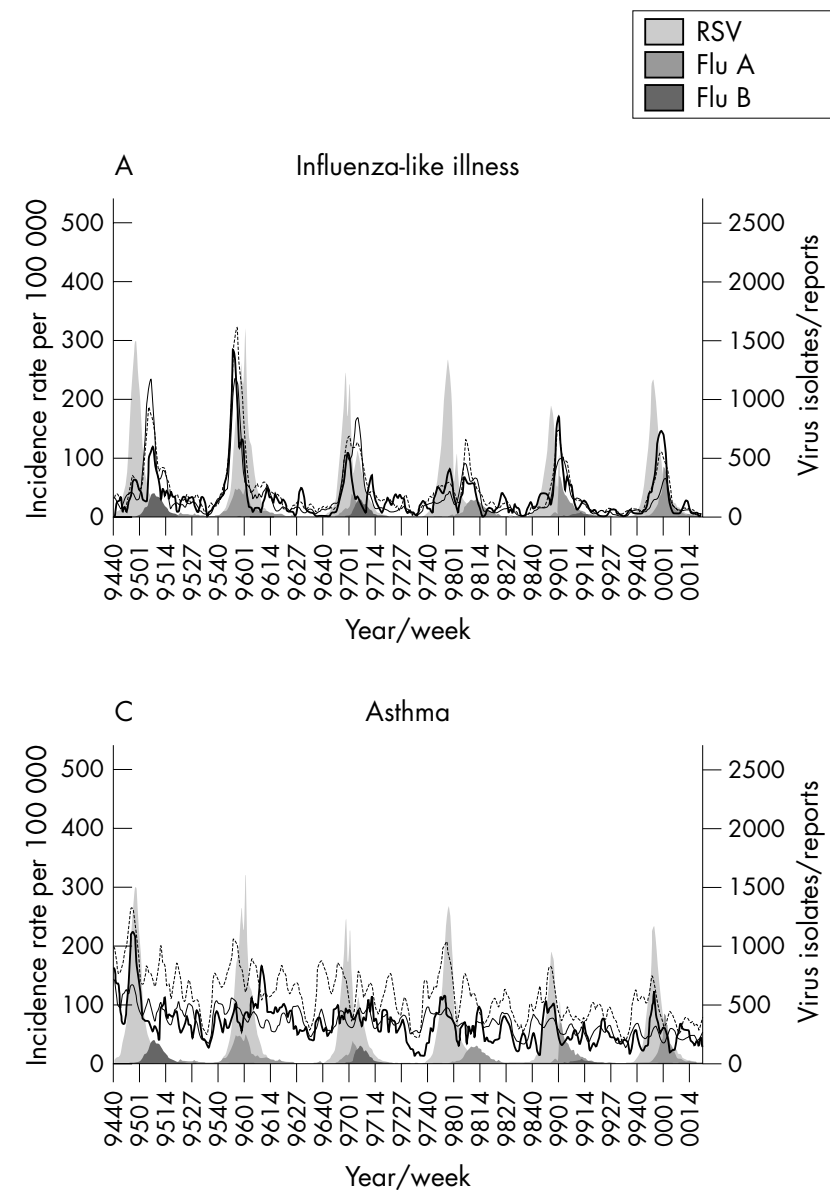

\begin{tabular}{c}
$-<1$ year \\
$1-4$ years \\
$-5-14$ years \\
\hline
\end{tabular}
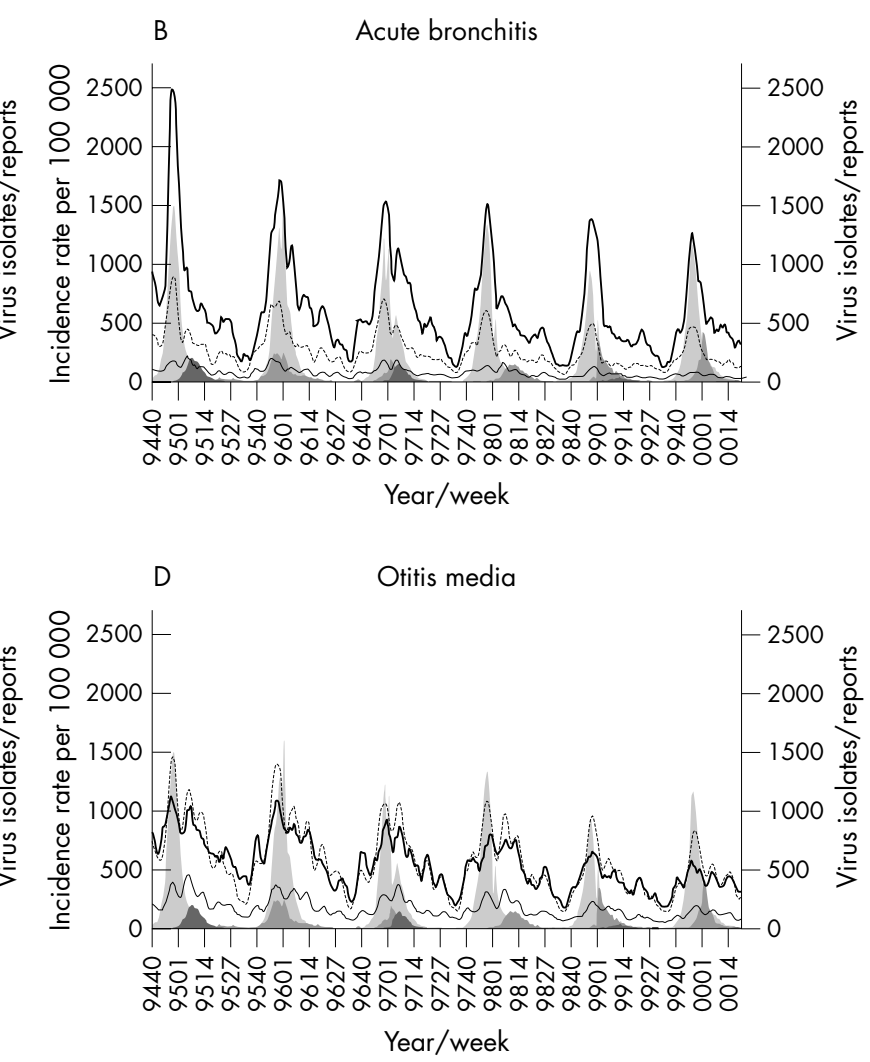

Figure 1 Weekly incidence rate per 100000 persons of (A) influenza-like illness, (B) acute bronchitis, (C) asthma, and (D) otitis media compared with influenza virus isolates and respiratory syncytial virus (RSV) reports: three week moving average by age over years 1994 to 2000.

years and in the relative impact of each virus. For ILI, in each of the age groups, estimates for the influenza period exceed those in the RSV period in 1994/95, 1997/98 (except children $<1$ year), and 1998/99: rates were similar in remaining winters. For acute bronchitis (except 1995/96 and 1996/97), the estimates attributed to RSV are substantially higher than to influenza. For asthma, only in 1995/96 and 1997/98 was there any evidence of excess during influenza active periods. There were several negative estimates in the influenza active period, and taken as an average over the six winters, the incidence of asthma did not increase during influenza active periods. There were however substantial excesses of asthma in RSV periods every winter. For otitis media, cumulative winter excess rates in children $1-4$ years were slightly higher in RSV than in influenza virus active periods, but in other age groups they were similar.
In table 4 we report the relative proportions of excess diagnoses in each age group averaged over the six winters in the respective influenza virus and RS virus active periods, as calculated in the broad and narrow definitions of virus active period. For ILI in children less than 1 year (as an example), allocation of the estimate of excess ILI cases according to the broad definition ( $\mathrm{n}=30352$ over the six winters) was 54\% to influenza and $46 \%$ to RSV, but based on the narrow definition $(\mathrm{n}=23$ 990), the excess was distributed more towards influenza $(66 \% / 34 \%)$. As expected, the numerical estimates derived from the narrow definition were less than those derived from the broad definition (mostly 25-50\% less, data not presented). Using the narrow definition, the proportion of ILI attributed to influenza increased from $60 \%$ to $77 \%$, for acute bronchitis it decreased from $37 \%$ to $32 \%$, for asthma from $9 \%$ to $0 \%$, and for otitis media from $48 \%$ to $45 \%$. The narrow

Table 3 Average weekly baseline incidence (per 100 000) in virus non-active periods by diagnosis and age group

\begin{tabular}{|c|c|c|c|c|c|c|c|c|c|c|c|c|}
\hline \multirow[b]{2}{*}{ Year } & \multicolumn{3}{|l|}{ ILI } & \multicolumn{3}{|c|}{ Acute bronchitis } & \multicolumn{3}{|c|}{ Asthma } & \multicolumn{3}{|c|}{ Otitis media } \\
\hline & $<1$ & $1-4$ & $5-14$ & $<1$ & $1-4$ & $5-14$ & $<1$ & $1-4$ & $5-14$ & $<1$ & $1-4$ & $5-14$ \\
\hline $94 / 95$ & 24 & 36 & 20 & 596 & 258 & 77 & 100 & 150 & 92 & 664 & 605 & 180 \\
\hline $95 / 96$ & 34 & 44 & 33 & 600 & 282 & 83 & 98 & 132 & 73 & 699 & 698 & 214 \\
\hline $96 / 97$ & 21 & 27 & 19 & 590 & 253 & 71 & 74 & 128 & 71 & 565 & 529 & 157 \\
\hline $97 / 98$ & 13 & 28 & 21 & 474 & 236 & 73 & 54 & 103 & 73 & 528 & 551 & 155 \\
\hline $98 / 99$ & 20 & 20 & 18 & 361 & 147 & 49 & 39 & 90 & 58 & 379 & 382 & 114 \\
\hline $99 / 00$ & 10 & 11 & 10 & 410 & 165 & 42 & 43 & 79 & 47 & 387 & 388 & 111 \\
\hline
\end{tabular}



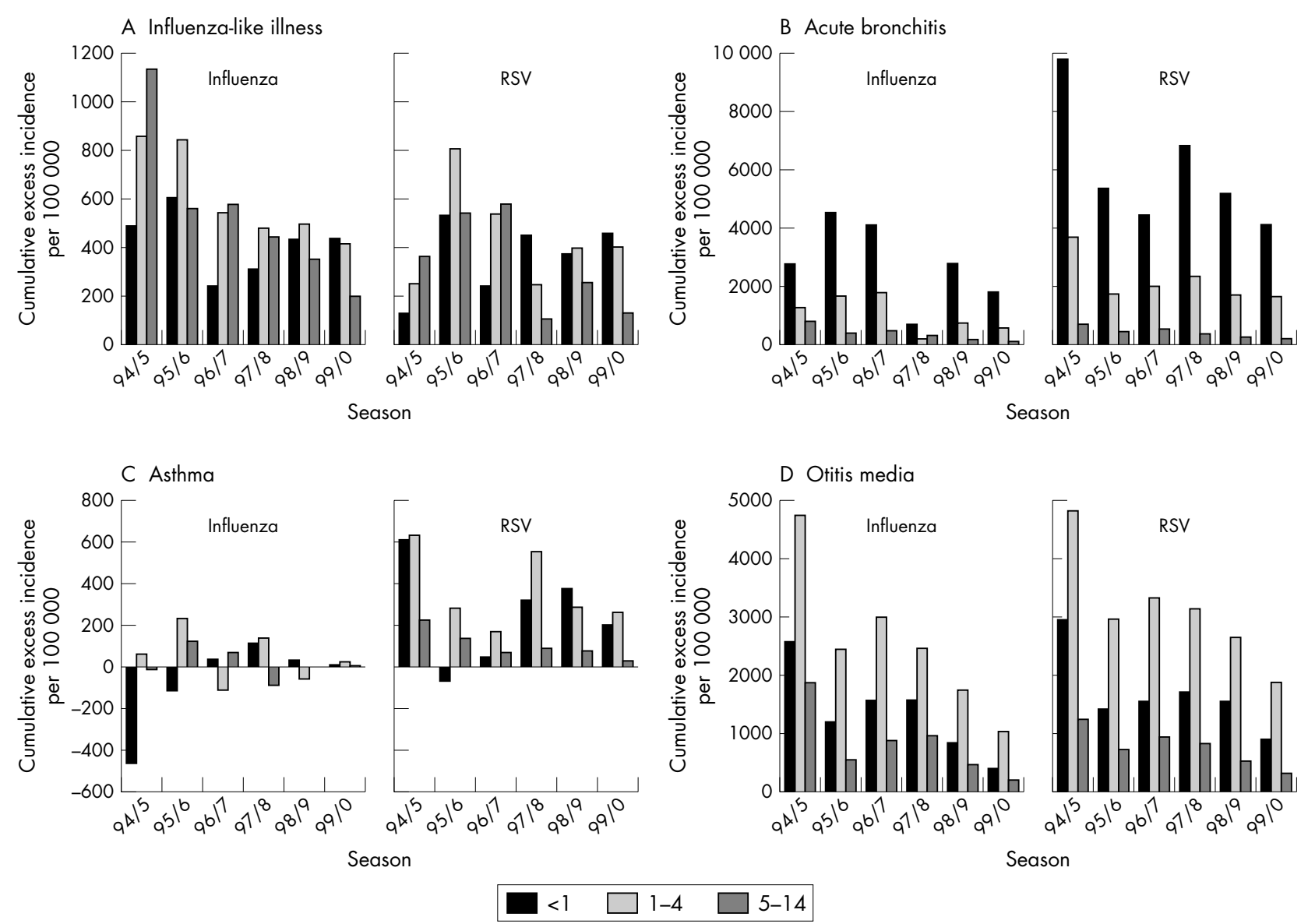

Figure 2 Cumulative excess incidence per 100000 of (A) influenza-like illness, (B) acute bronchitis, (C) asthma, and (D) otitis media, allotted to influenza and respiratory syncytial virus (RSV) active periods for age bands $<1,1-4$, and 5-14 years over winters 1994/95 to 1999/00.

definition increases the relative importance of RSV for all conditions except ILI. The estimates derived by using other definitions of virus active periods, including greater proportions of virus reports, were similar to those found for the broad definition.

\section{DISCUSSION}

The findings of this study suggest:

- The overall impact of RSV on respiratory illnesses in children of all ages exceeds that due to influenza.

- ILI is diagnosed more frequently in periods subsequently established as influenza active than those established as RSV active. However, there remain substantial numbers of cases of ILI reported in RSV active periods in all age groups.

- Excess incidence of acute bronchitis during periods of RSV activity in age groups $<1$, and $1-4$ years is twice that seen during influenza active periods: in children 5-14 years the excess incidence rates were similar.

- Asthma episodes occur much less frequently than acute bronchitis and are not increased during most influenza periods, whereas they are substantially increased in RSV periods in all three age groups.

- Otitis media is increased equally during RSV and influenza active periods. In children $1-4$ and 5-14 years, excess incidence exceeded that for each of the other diagnoses.

\begin{tabular}{|c|c|c|c|c|c|}
\hline & & \multicolumn{4}{|c|}{ Influenza/RSV \% of total } \\
\hline & & $<1$ years & $1-4$ years & $5-14$ years & All ages \\
\hline \multirow[t]{2}{*}{ Influenza-like illness } & Broad & $54 / 46$ & $58 / 42$ & $62 / 38$ & $60 / 40$ \\
\hline & Narrow & $66 / 34$ & $72 / 28$ & $80 / 20$ & $77 / 23$ \\
\hline \multirow[t]{2}{*}{ Acute bronchitis } & Broad & $32 / 68$ & $32 / 68$ & $49 / 51$ & $37 / 63$ \\
\hline & Narrow & $26 / 74$ & $26 / 74$ & $48 / 52$ & $32 / 68$ \\
\hline \multirow[t]{2}{*}{ Asthma } & Broad & $0 / 100^{*}$ & $12 / 88$ & $12 / 88$ & $9 / 91$ \\
\hline & Narrow & $0 / 100^{*}$ & $0 / 100^{*}$ & $0 / 100^{*}$ & $0 / 100^{*}$ \\
\hline \multirow[t]{2}{*}{ Otitis media } & Broad & $45 / 55$ & $45 / 55$ & $52 / 48$ & $48 / 52$ \\
\hline & Narrow & $43 / 57$ & $41 / 59$ & $51 / 49$ & $45 / 55$ \\
\hline
\end{tabular}




\section{What is already known on this topic}

- Influenza and respiratory syncytial virus are well recognised causes of regular winter respiratory infections

- The relative impact of each is unclear

- The incidence of respiratory infections during winter outside the periods of influenza and RSV activity declined over the six winters as has been reported previously from WRS data based on all ages. ${ }^{20}$ Influenza and RS viruses are highly variable from year to year and these cannot usefully be evaluated for trend in a limited six winter period.

The conclusions relating to the impact of influenza and RS virus are reinforced by the analysis using the narrow definition of active periods to try and separate their impact. The implications of these findings can be considered in two areas: clinical diagnosis and vaccination policy.

\section{Clinical diagnosis}

There is always considerable variability in operational research using general practice diagnoses unsubstantiated in individual cases by virological investigation. However, this study is comparing the effects of RSV and influenza in a clinical environment in which there is no selective bias in favour of one organism or the other. It was of particular interest to note the relative precision of the diagnosis of ILI during influenza active periods and of acute bronchitis during RSV active periods. The importance of lower respiratory tract clinical signs in the diagnosis of these two conditions could perhaps be exploited to greater clinical effect in routine clinical practice, though the distinction can never be regarded as absolute. Influenza and RSV active periods were least separable in winters 1995/96 and 1996/97, creating the maximum difficulty for allotting excesses to each of the conditions separately. This fact probably affected the results for asthma: in the remaining winters excess incidence was more clearly associated with RSV active periods. In a study of elderly hospitalisations, Falsey et al noted increased likelihood of wheeze in RSV as opposed to influenza infection. ${ }^{21}$ Furthermore the well recognised syndrome of bronchiolitis in young children is associated with lower respiratory tract clinical signs and mostly due to RSV.

In the analysis based on the broad definition of influenza virus and RS virus active periods, the cumulative excess incidence of all diagnoses attributed to both influenza and RSV exceeded those based on the narrow definition. Using either definition, estimates of excess incidence of otitis media in age groups 1-4 and 5-14 years slightly exceeded those for acute bronchitis and both substantially exceeded those for ILI and asthma. In children $<1$ year, estimates of the excess incidence of acute bronchitis exceeded the others.

\section{Vaccination policy}

These results emphasise the need for effective vaccines and treatments against disease caused by RSV. They further strongly support the inclusion of otitis media and of acute bronchitis as important end points in the evaluation of vaccine efficacy and effectiveness studies for interventions involving both viruses. ${ }^{22-24}$ Current NHS policy in the UK recommends that asthmatic children aged 2 years and over should be vaccinated against influenza. The basis of this policy stems largely from the accumulated evidence from adults with asthma and chronic obstructive pulmonary

\section{What this study adds}

- RSV is more important than influenza as a cause of respiratory infection in children $<1$ year and 1-4 years

- In children 5-14 years the overall impact of each virus was virtually the same

- Excess morbidity diagnosed as asthma was found solely in RSV periods

- There were more excess episodes of otitis media during active virus periods than each of the other conditions, and almost equal proportions were attributable to influenza and RSV

disease. ${ }^{2}$ Viral respiratory infections, generally though not influenza specifically, are recognised as a major cause of exacerbation of asthma. ${ }^{12}{ }^{13}$ Our findings challenge the need for routine vaccination of healthy children because of the risk of asthma attacks occurring as a complication of influenza infection. This is not to be confused with the vaccination of asthmatic children; though, with no increased asthma detected during influenza active periods there would appear little likelihood of increased asthma attacks in asthmatic children. Seasonal patterns of episodes of asthma in children presenting to general practitioners as reported in the WRS are very similar to patterns of hospital admission for asthma. ${ }^{10}$ Our results accord with the findings of Bueving et al who studied asthma in a randomised trial of influenza vaccine in asthmatic children aged 6-18 years in 1999/00 and 2000/01; and with the Cochrane review of the benefits of influenza vaccination in childhood asthmatics. ${ }^{125}$

\section{ACKNOWLEDGEMENTS}

We gratefully acknowledge the contribution of the WRS sentinel practices and their staff to providing the GP episode data. The Birmingham Research Unit of the Royal College of General Practitioners is funded by the Department of Health.

\section{Authors' affiliations}

D M Fleming, R S Pannell, A J Elliot, K W Cross, Birmingham Research Unit of The Royal College of General Practitioners, Birmingham, UK

Competing interests: none

\section{REFERENCES}

1 Cates CJ, Jefferson TO, Bara Al, et al. Vaccines for preventing influenza in people with asthma. Cochrane Database Syst Rev 2004:CD000364.

2 Harper SA, Fukuda K, Uyeki TM, et al. Prevention and control of influenza: recommendations of the Advisory Committee on Immunization Practices (ACIP). MMWR Recomm Rep 2004;53:1-40.

3 Collier L, Oxford J. Childhood infections caused by paramyxoviruses. Human virology. A text for students of medicine, dentistry and microbiology. Oxford: Oxford Medical Publications, 1996:117-18.

4 Treanor JJ, Fleming DM. Respiratory viruses. In: Galasso GJ, ed. Practical guidelines in antiviral therapy. Elsevier, 2002:223-56.

5 Nicholson KG. Human influenza. In: Nicholson KG, Webster RG, Hay AJ, eds. Textbook of influenza. Oxford: Blackwell Science, 1998:219-64.

6 Isaacs D, Joshi P. Respiratory infections and asthma. Med J Aust 2002;177:S50-1.

7 Message SD, Johnston SL. Viruses in asthma. Br Med Bull 2002;61:29-43.

8 Peebles RS Jr. Viral infections, atopy, and asthma: is there a causal relationship? J Allergy Clin Immunol 2004;113:S15-18.

9 Tuffaha A, Gern JE, Lemanske RF Jr. The role of respiratory viruses in acute and chronic asthma. Clin Chest Med 2000;21:289-300.

10 Fleming DM, Cross KW, Sunderland R, et al. Comparison of the seasonal patterns of asthma identified in general practitioner episodes, hospital admissions, and deaths. Thorax 2000;55:662-5.

11 Azevedo AM, Durigon EL, Okasima V, et al. Detection of influenza, parainfluenza, adenovirus and respiratory syncytial virus during asthma attacks in children older than 2 years old. Allergol Immunopathol (Madr) 2003;31:311-17. 
12 Johnston SL. Influence of viral and bacterial respiratory infections on exacerbations and symptom severity in childhood asthma. Pediatr Pulmono Suppl 1997;16:88-9

13 Nicholson KG, Kent J, Ireland DC. Respiratory viruses and exacerbations of asthma in adults. BMJ 1993;307:982-6.

14 Peiris JS, Tang WH, Chan KH, et al. Children with respiratory disease associated with metapneumovirus in Hong Kong. Emerg Infect Dis 2003;9:628-33.

15 Thumerelle C, Deschildre A, Bouquillon C, et al. Role of viruses and atypical bacteria in exacerbations of asthma in hospitalized children: a prospective study in the Nord-Pas de Calais region (France). Pediatr Pulmonol 2003;35:75-82.

16 Fleming DM, Sunderland R, Cross KW, et al. Declining incidence of episodes of asthma: a study of trends in new episodes presenting to general practitioners in the period 1989-98. Thorax 2000;55:657-61

17 Fleming DM. A perspective from general practice on selected influenza topics: near patient tests, influenza in pregnancy, influenza in children. In: Potter CW ed. Influenza. Amsterdam: Elsevier, 2002:123-44.

18 Fleming DM. Weekly Returns Service of the Royal College of General Practitioners. Commun Dis Public Health 1999;2:96-100.
19 Fleming DM, Zambon M, Bartelds Al, et al. The duration and magnitude of influenza epidemics: a study of surveillance data from sentinel general practices in England, Wales and the Netherlands. Eur J Epidemiol 1999:15:467-73.

20 Fleming DM, Ross $\mathrm{AM}$, Cross $\mathrm{KW}$, et al. The reducing incidence of respiratory tract infection and its relation to antibiotic prescribing. Br J Gen Pract 2003;53:778-83

21 Falsey AR, Cunningham CK, Barker WH, et al. Respiratory syncytial virus and influenza $A$ infections in the hospitalized elderly. J Infect Dis 1995; 172:389-94.

22 Glezen WP. Prevention of acute otitis media by prophylaxis and treatment of influenza virus infections. Vaccine 2000;19:S56-8.

23 Heikkinen T, Thint $M$, Chonmaitree T. Prevalence of various respiratory viruses in the middle ear during acute otitis media. N Engl J Med 1999:340:260-4.

24 Hoberman A, Greenberg DP, Paradise JL, et al. Effectiveness of inactivated influenza vaccine in preventing acute otitis media in young children: a randomized controlled trial. JAMA 2003;290:1608-16.

25 Bueving HJ, Bernsen RM, de Jongste JC, et al. Influenza vaccination in children with asthma: randomized double-blind placebo-controlled trial. Am J Respir Crit Care Med 2004; 169:488-93. 